\title{
Studio preliminare sulle oscillazioni libere del Golfo di Cagliari
}

\author{
P. CAloi - M. C. SPAnEA \\ Ricevuto il 1 Febbraio 1962
}

Proseguendo lo studio sull'idrodinamica dei golfi interessanti l'Italial, abbiamo questa volta preso in esame il golfo di Cagliari (Fig. 1).

Il golfo di Cagliari sito nella parte meridionale della Sardegna, si apre, con un'ampia bocea che dal Capo Pula va al Capo Carbonara lungo una linea ideale di $45 \mathrm{~km}$ circa, sul Mar Mediterraneo. Caratteristica di questo golfo è la eccezionale larghezza della sua bocca rispetto alla sua lunghezza, che risulta essere di $22,5 \mathrm{~km}$.

1. - Oggetto della nostra nota è stato quello di determinare il periodo dell'uninodale dell'intero golfo. Si è suddiviso, a tale scopo, il golfo suddetto in 22 sezioni; di queste si sono calcolate le aree verticali e in superficie (Fig. 2). Ottenuti questi dati, ci è stato possibile applicare il metodo di Goldberg, per la descrizione del quale rimandiamo ad altra nota $\left(^{1}\right)$. Dopo vari tentativi si è trovato, per il periodo dell'uninodale, un valore di

$$
\mathrm{T}=10^{\mathrm{m}}, 2
$$

valore che conduce ad un residuo trascurabile. Poichè, come abbiamo già detto, l'ampiezza della bocea del golfo è molto rilevante, ej si doveva attendere una correzione molto elevata, conformemente del resto alla teoria ${ }^{2}{ }^{2}$; infatti il fattore più rispondente alle osservazioni è risultato essere quello di 1,5: ne consegue per il periodo corretto un valore di

$$
\mathrm{T}=60^{\mathrm{m}}=1^{\mathrm{h}}
$$

in accordo con quello osservato sui mareogrammi, ottenuti dal mareografo sito presso la Capitaneria del porto di Cagliari.

È da aggiungere inoltre che, nelle osservazioni mareografiche, fral le altre, sono state isolate e messe in evidenzal numerose oscillazioni con un 
periodo di circa 2$)^{\mathrm{m}}$, periodo questo che dovrebbe corrispondere a quello della binodale dell'intero golfo.

Nella Tabella I sono riportati i dati ottenuti per l'applicazione del metodo di Golrlberg.

Tabella I

\begin{tabular}{|c|c|c|c|c|c|c|}
\hline $\begin{array}{l}\text { Se- } \\
\text { zioni }\end{array}$ & $\begin{array}{c}\Delta(\mathrm{x})_{v} \\
\mathrm{~m}\end{array}$ & $\begin{array}{c}S(\mathbf{x})_{y} \\
10^{6} \mathrm{~m}^{2}\end{array}$ & $\begin{array}{l}\Delta v(\mathrm{x})_{v} \\
10^{8} \mathrm{~m}^{2}\end{array}$ & $\begin{array}{l}\xi_{\nu} \\
\mathrm{m}\end{array}$ & $\begin{array}{l}\eta_{\nu} \\
\mathrm{m}\end{array}$ & $\begin{array}{c}m_{\nu} \\
10^{12} \mathrm{~m}^{3}\end{array}$ \\
\hline 0 & & 12.157 & 0 & 82.257 & 0 & 1,000 \\
\hline 1 & 1.000 & 11.146 & 45,25 & 89.629 & 56,8396 & 0,999 \\
\hline 2 & $n$ & 9,488 & 42,40 & 104.975 & 118,7732 & 0,996 \\
\hline 3 & $"$ & 8.203 & 42,18 & 120.931 & 191,3109 & 0,992 \\
\hline 4 & $n$ & 6.556 & 42,69 & 150.396 & 274,8742 & 0,986 \\
\hline 5 & $"$ & 5,477 & 39,54 & 178.747 & 378,7978 & 0,979 \\
\hline 6 & $"$ & 4,878 & 32,87 & 199.057 & 502,3120 & 0,971 \\
\hline 7 & $"$ & 3,864 & 39,51 & 247.930 & 639,8604 & 0,958 \\
\hline 8 & $"$ & 3,554 & 28,62 & 266.179 & 811,1800 & 0,946 \\
\hline 9 & $"$ & 3,371 & 27,58 & 276.476 & 995,1097 & 0,932 \\
\hline 10) & $"$ & 2,226 & 29,40 & 411.051 & $1.186,1546$ & 0,915 \\
\hline 11 & $"$ & 1,294 & 29,21 & 690.881 & $1.470,1908$ & 0,894 \\
\hline 12 & $"$ & 0,996 & 27,49 & 870.482 & $1.947,5896$ & 0,867 \\
\hline 13 & $"$ & 0,889 & 25,12 & 939.258 & $2.549,0927$ & 0,835 \\
\hline 14 & " & 0,706 & 27,64 & 1.120 .397 & $3.198,1200$ & 0,791 \\
\hline 15 & $"$ & 0,646 & 25,02 & 1. 147.059 & $3.972,3143$ & 0,741 \\
\hline 16 & $"$ & 0,468 & 27,25 & 1.444 .444 & $4.764,9321$ & 0,676 \\
\hline 17 & $"$ & 0,383 & 26,90 & 1.561 .358 & $5.763,0429$ & 0,598 \\
\hline 18 & $"$ & 0,343 & 25,70 & 1.486 .880 & $6.841,9413$ & 0,510 \\
\hline 19 & " & 0,271 & 35,67 & 1.365 .314 & $7.869,3754$ & 0,370 \\
\hline 20 & $"$ & 0,228 & 22,14 & 1. 144.737 & $9.812,8074$ & 0,261 \\
\hline 21 & » & 0,136 & 28,77 & 794.118 & $10.603,8207$ & 0,108 \\
\hline 22 & 750 & & 17,29 & & $11.015,3719$ & 0,013 \\
\hline
\end{tabular}

2. - Al fine di selezionare le oscillazioni dovute esclusivamente al golfo, nella sua totalità, da quelle proprie del porto di Cagliari e delle sue suddivisioni, sarebbe necessario poter registrare anche ad oriente di Capo S. Elia. Le registrazioni ottenute dal mareografo sito presso la Capitaneria del porto di Cagliari, contengono le oscillazioni libere non solo dell'intero golfo e dello zoccolo dell'isola, ma anche quelle derivanti dai bacini formati dai numerosi moli che dividono il porto di Cagliari propriamente detto in tante parti animate da oscillazioni proprie. Data la complessitì di queste suddivisioni, alcune delle quali entrano come parte integrante di quelle più estese, riesce molto difficile valutarne le caratteristiche dinamiche singole dal punto di vista teorico.

Il problema ì reso più arduo dai bassi fondali, che consentono un'azione ritardatrice della viscositì $\left({ }^{3}\right)$, non agevolmente valutabile. 


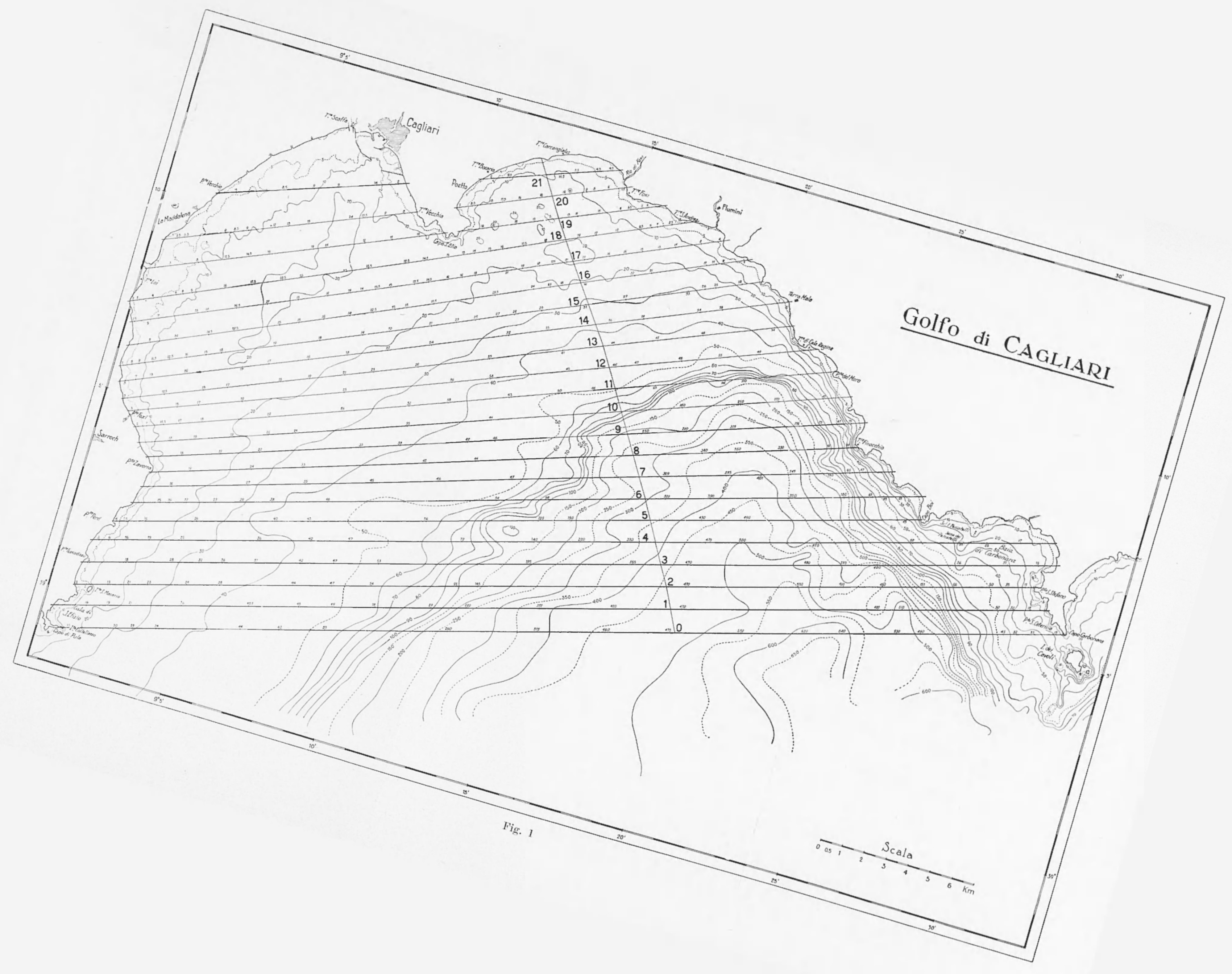




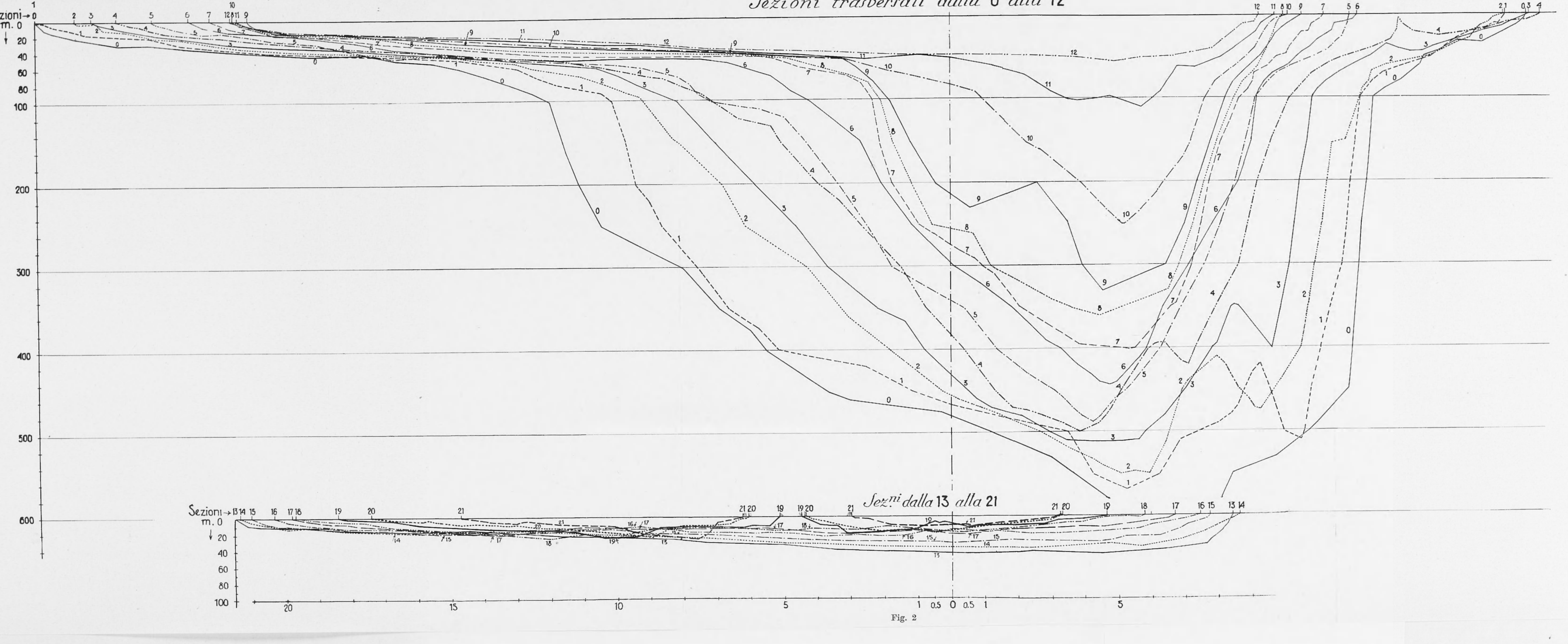




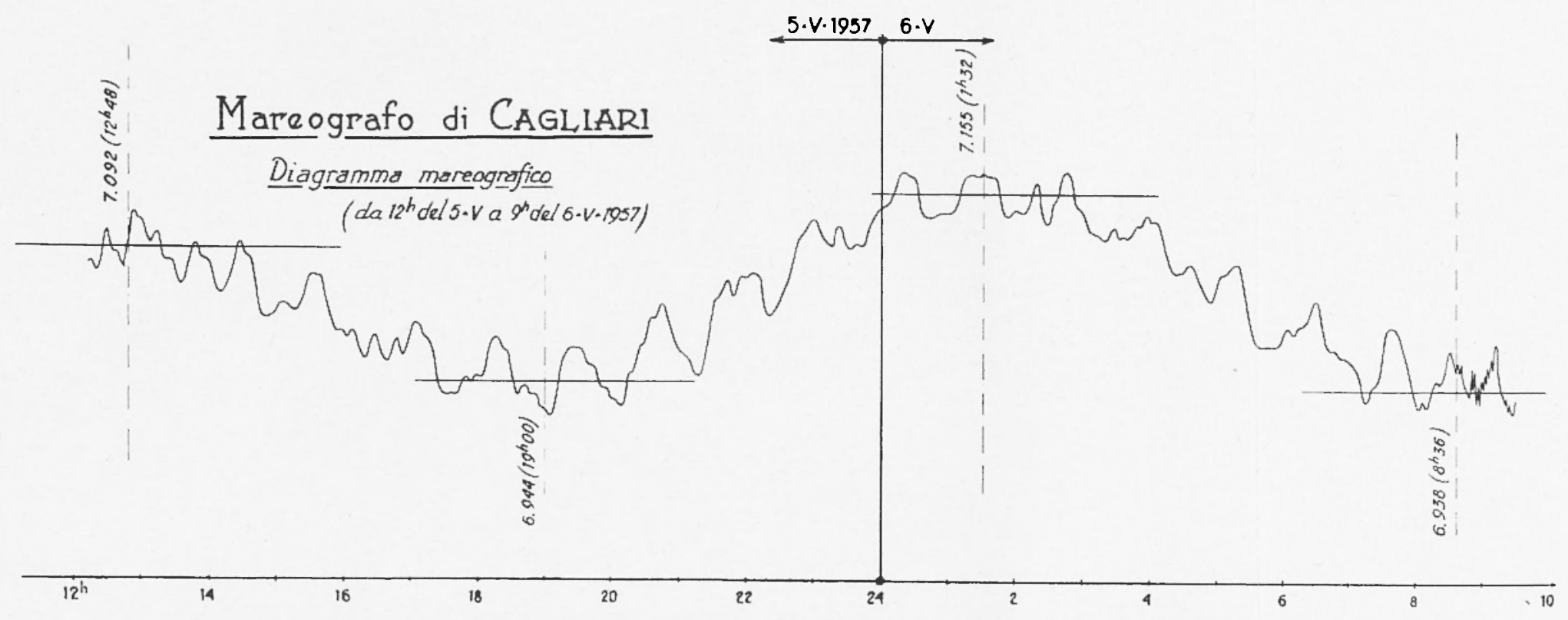

Fig. 3 - Esempi di oseillazioni libere uninodali (ridotte a circa $1 / 10$ dell'ampiezza naturale) 

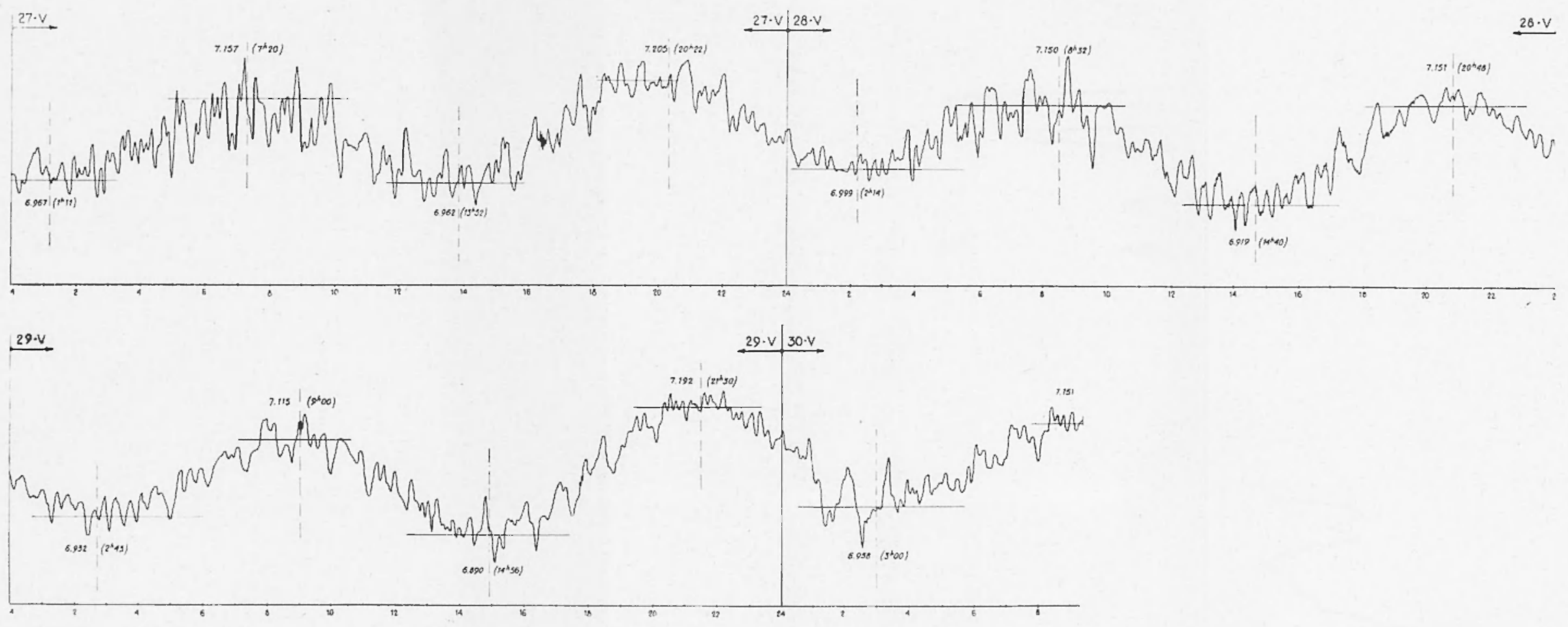

Fig. 4 - Esempio di oscillazioni libere uni e binodali dell'intero golfo e di sue parti (ridotti a circa l/15 dell'ampiezza naturale) 

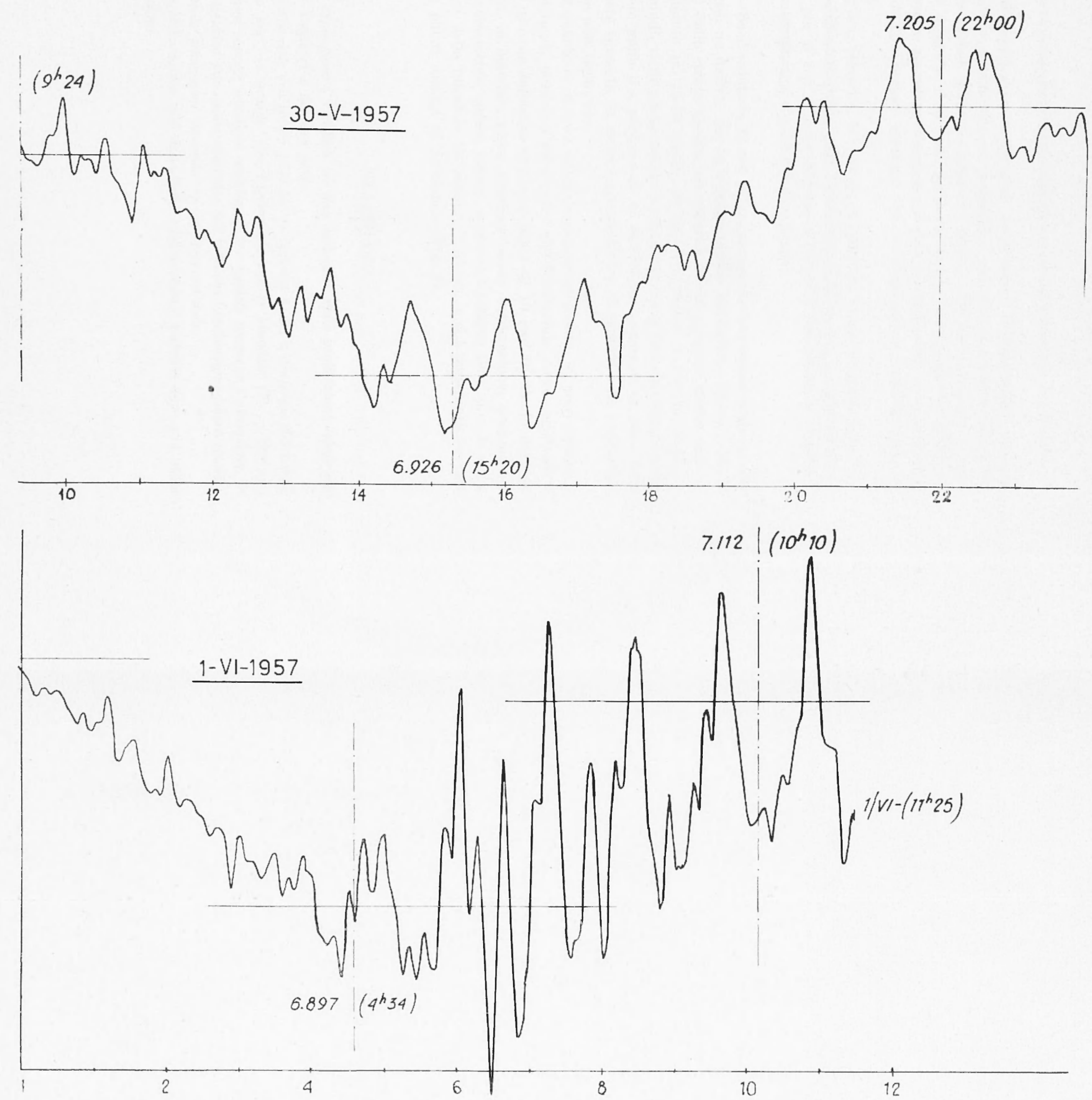

Fig. 5 - Chiari esempi di oscillazioni libere uninodali e binodali (ridotti a $1 / 5$ dell'ampiezza naturale) 
Eventuali oscillazioni multinodali interessanti l'intero golfo, possono quindi essere mascherate da oscillazioni associate dai bacini del porto. Di qui la grande utilità - come si ̀̀ detto - di poter usufruire di registrazioni ottenute al di fuori del porto stesso. Solo in questo modo potrebbe essere agevolato il compito di una piu attendibile interpretazione dei molteplici periodi figuranti fra le registrazioni mareografiche di Cagliari.

Per ora, pertanto, la nostra indagine può essere limitata soltanto a quelle oscillazioni per le quali l'interpretazione sembra accettabile anche perché, per il suo completamento, si renderà necessario il confronto con i contemporanei elementi meteorologici.

3. - Se si valuta in $13 \mathrm{~km}$ la lunghezza medio-massima dello zoccolo sommerso, ne deriva, per la corrispondente oscillazione libera ("Shelfseiche ") dello strato liquido sovrastante (il cui spessore medio può essere valutato sui $15-20$ metri), un periodo proprio di $65^{\mathrm{m}} \mathrm{ca}$. Tale periodo quindi, viene praticamente a coincidere con il periodo fondamentale dell'intero golfo. La persistenza di oscillazioni superanti di poco l'ora può essere spiegata da questa concomitanza di periodi e da eccitazioni prossime alla risonanzil.

Per quanto si riferisce all'ampiezza, tenuto conto del poco materiale a disposizione, possiamo aggiungere che l'uninodale viene registrata a Cagliari con un'ampiezza medio-massima di $10 \mathrm{~cm}$. In alcuni dei casi osservati, le binodali, meno frequenti come registrazione, possono superare nettamente questo valore: il giorno 1 Giugno 1957 , p. e. la semiampiezza della binodale ha superato i $30 \mathrm{~cm}$., e si è presentata sovrapposta a chiari esempi di uninodale (Fig. 5).

\section{RIASSUNTO}

Si espongono $i$ risultati di una indagine sulle oscillazioni libere del golfo di Cagliari e del suo porto.

$L^{\prime}$ uninodale dell'intero golfo ha un periodo di $1^{h}$, a conferma del valore calcolato per via teorica. Sia l'uninodale che la binodale $\left(T=20^{\mathrm{m}}\right.$ ca.) presentano sovente sensibili ampiezze; per quanto concerne l'uninodale, la sua persistenza può essere agevolata dal fatto che l'oscillazione libera relativa allo zoccolo sommerso presenta un analogo periodo.

Un'elaborazione piu dettagliata sarà possibile soltanto dopo piu estese osservazioni. 


\section{$S U M M A R Y$}

The results of a first research on the free oscillations in the gulf of Cagliari and its port are exposed.

The uninodal of the entire gulf has a $1^{\text {h }}$ period, and so confirms the theoretically calculated value. The uninodal and the binodal $\left(T=20^{\mathrm{m}}\right.$ ca.) often show considerable amplitudes: for the uninodal, its persistence could be facilitated by the fact that the shelf-seich shows a similar period.

A more detailed elaboration will only be possible after more extensive observations.

\section{BIBLIOGRAFIA}

(') Calor P., Le sesse del Lago di Garda, Parte II, "Annali di Geofisica ", 1, 2, (1948).

(2) Calor P., Spadea M. C., Le sesse del Lago di Garda. Parte IV, "Annali di Geofisica ", VI, 4, (1953).

(3) Calor P., Spadea M. C., Le oscillazioni libere del golfo di Civitavechia e l'azione della viscosità cinematica, "Annali di Geofisica ", XII, 2, (1959). 